\title{
Evaluation of Th9 lymphocytes in peripheral blood of rheumatoid arthritis patients and correlation with anti-tumor necrosis factor therapy: results from an in vitro pivotal study
}

\author{
R. Talotta ${ }^{1}$, A. Berzi ${ }^{2}$, F. Atzeni' ${ }^{1}$, D. Dell'Acqua'1 , P. Sarzi Puttini', D. Trabattoni² \\ ${ }^{1}$ Rheumatology Unit; ${ }^{2}$ Department of Biomedical and Clinical Sciences, \\ University Hospital Luigi Sacco, University of Milan, Milan, Italy
}

\begin{abstract}
SUMMARY
The aim of this study was to determine the prevalence of T helper 9 (Th9) lymphocytes in rheumatoid arthritis (RA) patients and to identify a possible association between the percentage of Th9 and the discontinuation of a biological treatment with an anti-tumor necrosis factor (TNF) (infliximab).

We collected peripheral blood mononuclear cells (PBMCs) from 55 consecutive RA outpatients and 10 healthy controls. Among RA patients, 15 were not receiving any immunosuppressive drug, 20 were successfully treated with infliximab and 20 discontinued infliximab because of adverse events or inefficacy and were treated with other biological agents. PBMCs were cultured with/without infliximab $50 \mathrm{mg} / \mathrm{L}$ for $18 \mathrm{~h}$, and the percentage of Th9 cells was assessed by means of flow cytometry. Th9 lymphocytes were identified as interferon gamma, interleukin (IL)4-, IL17-, IL9-secreting cluster of differentiation 4 (CD4)+ T cells. Cytometric analysis revealed no significant decrease in the percentage of Th9 cells after infliximab exposure in any of the groups, although it was lower in healthy controls than RA patients either before and after the infliximab stimulation assay.

Th9 cells are IL-9-secreting T helper lymphocytes whose role in RA is still poorly known. IL-9 levels are increased in RA patients, in whom this cytokine plays a crucial role. Th9 cells are the major producers of IL-9, and their prevalence is higher in RA patients than in healthy subjects; however our experiment in vitro does not demonstrate an association between Th9 lymphocytes and the response to infliximab. Further studies are required to evaluate the real involvement of Th9 population in the immunogenicity of anti-TNF agents.
\end{abstract}

Key words: Infliximab; immunogenicity; Th9 lymphocytes; rheumatoid arthritis.

Reumatismo, 2016; 68 (2): 83-89

\section{INTRODUCTION}

heumatoid arthritis (RA) is a chronic autoimmune disease affecting up to $1 \%$ of worldwide population with a progressively disabling course. RA is characterized by chronic synovitis of peripheral joints, mainly ruled by the uncontrolled activation of cells belonging to the adaptive immunity.

Cluster of differentiation 4 (CD4)+ T lymphocytes play a central role in the induction and in the maintenance of the disease, due to their complex interplay with dendritic cells, macrophages, and B-lymphocytes. According to the cytokine milieu, CD4+ $\mathrm{T}$ lymphocytes may differentiate into distinct phenotypes, each of which plays a precise role in inducing, tuning and repressing the immune response. Over the past twenty years, new acquisitions in the immunologic field led to the definition of five distinct $\mathrm{T}$ helper (Th) cell populations: Th1 cells mainly secreting interferon gamma (IFN $\gamma$-), Th2 cells mainly secreting interleukin (IL)-4, Th17 cells secreting IL-17 and involved in autoimmune diseases, Th22 cells secreting IL-22 and Th9 cells secreting IL-9 (1). A counterpart of $\mathrm{T}$ cells with repressive properties, named $\mathrm{T}$ regulatory cells (Tregs), was also described. In RA, T helper lymphocytes mostly give rise to Th2 or Th17 responses (2). Th9 are a novel pool of T $\overline{\text { Corresponding author }}$

Rossella Talotta

Rheumatology Unit, University Hospital Luigi Sacco Via Gian Battista Grassi, 74 20157 Milan, Italy E-mail: talotta1@virgilio.it 
helper lymphocytes, almost implied in allergic diseases. There is little knowledge about the role of Th9 in RA. Indeed, IL-9 is increased in serum and synovial fluid of RA patients. A recent study demonstrated that Th9 cells are responsible for the degree of lymphoid organisation in the synovial membrane of RA patients, being also related to the titre of anti-cyclic peptide antibodies (ACPAs) (3). However, due to their peculiar property of orchestrating both Th17 and Tregs responses, Th9 cells own a more complex function in the immunological network at the basis of RA. Furthermore, no study has still attempted to clarify the implication of these cells in the response to a biological treatment with an anti-tumor necrosis factor (TNF) agent. In a previous study on RA patients (4) we demonstrated that the stimulation in vitro of peripheral blood mononuclear cells (PBMCs) with infliximab could induce a paradoxical activation of Th1 and Th17 lymphocytes while repressing $\mathrm{T}$ regulatory cells in those patients who had failed the treatment with infliximab. Accordingly, we hypothesized that Th9 lymphocytes may vary in response to infliximab, conditioning the final outcome of the therapy. The primary objective of our study was therefore to evaluate the prevalence of Th9 lymphocytes in the peripheral blood of RA patients compared with a group of matched healthy controls.

The secondary objective was to detect the association between the percentages of Th9 cells and the clinical response to a TNFblocker (infliximab), following a stimulation test in vitro.

\section{MATERIALS AND METHODS}

We enrolled 55 consecutive RA outpatients diagnosed according to the American College of Rheumatology/European League Against Rheumatism (ACR/EULAR) 2010 criteria (5). Patients were recruited from our previous study (4). This cohort included 15 subjects affected by early arthritis not concurrently treated with immunosuppressive drugs, 20 patients successfully treated with infliximab, 20 patients who had swapped to biologic drugs other than infliximab due to adverse events or inefficacy, and a matched control group of 10 healthy subjects.

Demographic characteristics are resumed in Table I. Patients were concomitantly treated with prednisone $\leq 10 \mathrm{mg} /$ day (at a stable dose within 4 weeks since blood sampling); methotrexate $\leq 15 \mathrm{mg} /$ week, sulfasalazine $\leq 3 \mathrm{~g} /$ day, hydroxychloroquine $\leq 400 \mathrm{mg} /$ day, all provided that were administered at stable dose in the 6 weeks before the blood sample. In the group of non responder, the patients were treated with intravenous (i.v.) abatacept $10 \mathrm{mg} /$ kg every 4 weeks (13 subjects), i.v. tocilizumab $8 \mathrm{mg} / \mathrm{kg}$ every 4 weeks (5 subjects), subcutaneous (s.c.) etanercept $50 \mathrm{mg}$ once a week (1 subject), or s.c. certolizumab pegol $200 \mathrm{mg}$ every other week (1 subject), as second ( 8 patients), third ( 8 patients) or fourth biologic line (4 patients). Patients with concomitant infections, atopic dermatitis, haematological disorders, treated with leflunomide or cyclosporine, or receiving vaccinations in the previous 2 months were excluded. The study was approved by a local Ethical Committee and all the procedures were performed in accordance with the Declaration of Helsinki.

After giving an informed consent, peripheral blood from each subject was collected into Vacutainer tubes containing EDTA (Becton Dickinson; Rutherford, NJ, USA). PBMCs were isolated by centrifugation on lymphocyte separation medium (Cedarlane Laboratories, Burlington, NC, USA). The number and viability of PBMCs were determined by an automatic cell counter, ADAM-MC (Digital-Bio, NanoEnTek Inc., Seoul, Korea). PBMC viability was typically $>98 \%$.

Cell cultures were performed in RPMI 1640 plus penicillin, streptomycin, l-glutamine and $10 \%$ pooled Human AB Serum [all from Euroclone, Siziano (PV), Italy]. PBMCs, at a concentration of $1 \times 10^{6}$ cells/ $\mathrm{mL}$ were incubated for $18 \mathrm{~h}$ with culture medium alone or in the presence of $50 \mu \mathrm{g} /$ $\mathrm{mL}$ infliximab (Remicade, Janssen Biologics, Leiden, Netherlands), or $50 \mu \mathrm{g} / \mathrm{mL}$ recombinant Human IgG Fc (R\&D Systems, Minneapolis, MN, USA). Pulse-width mod- 
Table I - Demographic characteristics of the study population.

\begin{tabular}{|l|l|l|l|l|}
\hline Variables & $\begin{array}{l}\text { Healthy } \\
\text { controls }\end{array}$ & $\begin{array}{l}\text { Treatment-naïv } \\
\text { RA patients }\end{array}$ & $\begin{array}{l}\text { RA patients responding } \\
\text { to IFX }\end{array}$ & $\begin{array}{l}\text { RA patients not } \\
\text { responding to IFX }\end{array}$ \\
\hline No. & 10 & 15 & 20 & 20 \\
\hline Mean age \pm SD, years & $43.9 \pm 8.3$ & $54.8 \pm 16.2$ & $61.3 \pm 12.2$ & $57.0 \pm 12.2$ \\
\hline Mean disease duration \pm SD, years & $/$ & $2.3 \pm 3.9$ & $13.4 \pm 7.2$ & $18.1 \pm 9.5$ \\
\hline F/M & $4 / 6$ & $12 / 3$ & $16 / 4$ & $15 / 5$ \\
\hline ACPA+ & $/$ & 5 & 15 & 15 \\
\hline RF+ & $/$ & 7 & 11 & 15 \\
\hline ANA+ & $/$ & 2 & 12 & 17 \\
\hline Anti-dsDNA Ab+ & $/$ & 0 & 3 & 2 \\
\hline Anti-ENA Ab+ & $/$ & 0 & 1 & 3 \\
\hline ACLA/LAC+ & $/$ & 0 & 1 & 2 \\
\hline $\begin{array}{l}\text { Prednisone } \\
(2.5-10 \text { mg/day) }\end{array}$ & $/$ & $/$ & 8 & 14 \\
\hline $\begin{array}{l}\text { Methotrexate } \\
(5-15 \text { mg/week) }\end{array}$ & $/$ & $/$ & 20 & 9 \\
\hline $\begin{array}{l}\text { Hydroxychloroquine } \\
(200-400 \text { mg/day) }\end{array}$ & $/$ & $/$ & 3 & 5 \\
\hline NSAlDs & $/$ & 14 & As needed & As needed \\
\hline
\end{tabular}

RA, rheumatoid arthritis; IFX, infliximab; SD, standard deviation; F, females; M, males; ACPA, anti-citrullinated-protein antibodies; RF, rheumatoid factor; ANA, anti-nuclear antibodies; anti-dsDNA, anti-double stranded DNA antibodies; anti-ENA, anti-extractable nuclear antigen antibodies; ACLA, anticardiolipin antibodies; LAC, lupus anticoagulant; NSAIDs, non-steroidal anti-inflammatory drugs.

ulation (lectin from Phytolacca americana; $1 \mu \mathrm{g} / \mathrm{mL}$; Sigma-Aldrich, St. Louis, MO, USA) was used as a positive control to evaluate the responsiveness of PBMCs. To facilitate co-stimulation, $1 \mu \mathrm{g} / \mathrm{mL}$ anti-human CD28 (R\&D Systems) was added to the cell cultures. Brefeldin A (10 $\mu \mathrm{g} / \mathrm{mL}$; Sigma-Aldrich) was added after the first three hours in order to inhibit cytokine secretion.

The percentage of Th9 lymphocytes was determined by flow cytometric analysis. Th9 lymphocytes were identified as IFN $\gamma$-, IL4-, IL-17-, IL-9 secreting CD4+ T cells (6). A concentration of infliximab of $50 \mu \mathrm{g} / \mathrm{mL}$ was chosen after setting a titration test with increasing concentrations of the drug and on the basis of the infliximab median serum concentrations one hour after infusion (peak serum concentration: 39.9-219.1 $\mu \mathrm{g} / \mathrm{mL}$ ) (7). The following monoclonal antibodies (mAbs) were used: CD4 PE-Cy7, IFN $\gamma$ (Beckman Coulter, Milan, Italy), IL17 PerCP-Cy5.5 (Biolegend, San Diego, CA, USA), IL-9 APC and IL-4 PE (R\&D Systems). PBMCs were incubated $15 \mathrm{~min}$ - utes with the mAbs for the detection of cell surface antigens and fixed with $1 \%$ paraformaldehyde (PFA). Then cells were permeabilized with Saponin (Sigma) and stained with the antibodies for the detection of intracellular cytokines. Following a 45-minute incubation in ice, the cells were fixed with $1 \%$ PFA.

Lymphocyte population was gated based on the basis of forward and side scatter properties, and further gated for CD4 expression; at least 20,000 events were acquired within the CD4 gate. The samples were acquired using a Gallios flow cytometer and data were analysed using Kaluza software (both Beckman Coulter).

As data were normally distributed, procedures were based on parametric analyses. Comparisons between the different groups were performed using unpaired Student's $t$ test for unequal variances with a two tailed $\mathrm{P}$ value. Significance was set at $\mathrm{P}<0.05$. Statistical analysis was performed using GraphPad Prism Software (GraphPad Software, San Diego, CA, USA). 


\section{RESULTS}

Our cohort included 20 consecutive RA patients (16 females; mean age 61.3 \pm 12.2 years; mean disease duration 13.4 \pm 7.2 years) successfully treated with infliximab $3 \mathrm{mg} / \mathrm{kg}$ every eight weeks, and 20 consecutive RA patients (15 females; mean age $57.0 \pm 12.2$ years; mean disease duration $18.1 \pm 9.5$ years) who discontinued infliximab due to inefficacy (11 cases) or adverse events (9 cases). Fifteen patients in both the two groups were positive for ACPAs, and respectively 11 responders and 15 non-responders were positive for rheumatoid factor (RF). During infliximab treatment, we detected anti-nuclear antibodies (ANAs) in 12 responders and 17 non-responders without any clinical involvement (Table I). The patients in both treated groups concomitantly received the allowed disease-modifying antirheumatic drugs and corticosteroids.

Cytometric analysis revealed no significant decrease in the percentage of Th9 cells after infliximab exposure in any of the groups, but percentage of Th9 cells was lower in healthy controls than RA patients

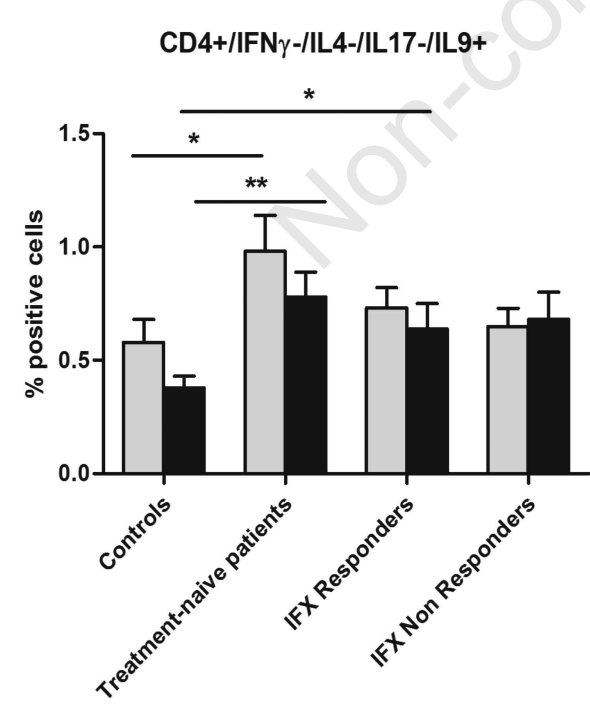

Figure 1 - The percentage of gamma interferon (IFN $\gamma$ )-, interleukin (IL)4-, IL17-, IL9-secreting CD4+ T cells in rheumatoid arthritis patients and healthy controls under unstimulated and infliximab (IFX)-stimulated conditions. Mean values \pm standard error. ${ }^{*} \mathrm{P}<0.05$; ${ }^{*} \mathrm{P}<0.01$ (Student's $t$ test). both before and after the stimulation assay with infliximab (Figure 1).

The higher frequency of Th9 cells in the patients was not associated with higher levels of anti-nucleus auto-antibodies or other auto-antibody subsets, or with a higher likelihood of experiencing an adverse event or lack of efficacy on infliximab treatment.

\section{DISCUSSION}

In our work we aimed firstly to evaluate the percentage of Th9 lymphocytes in peripheral blood of RA patients. Secondly, we performed an immune-stimulation assay in vitro in order to detect a possible relationship between Th9 cells and the outcome of a biologic therapy (infliximab). Th9 cell, discovered in 2008 by Veldhoen and colleagues, are a $\mathrm{T}$ helper cell subset developing from primary naïve $\mathrm{T}$ helper lymphocytes or from primed $\mathrm{T}$ helper 2 lymphocytes in presence of IL-4, TGF $\beta$, OX 40 and PU-1 (8). The main cytokine secreted is IL-9, but, in vitro, these cells may also produce IL-10, IL-17, IL-21 and IL-22 (9). It has been demonstrated that Th2 cells, Th17 cells, Treg cells, innate lymphoid cells, mast cells, natural killer cells are capable of releasing large amount of IL-9 (10). However, Th9 cells are a distinct class of CD4+ T lymphocytes, identified by a peculiar set of transcriptional factors (PU.1, IRF-4). The role played by Th9 cells has been investigated in vivo and in vitro. Th9 lymphocytes seem to be involved in the immunological responses underlying parasitic infections as well as allergic diseases $(11,12)$. A consistent number of reports has evidenced that these cells are highly represented in the airways of asthmatic subjects as well as in the skin of people suffering from atopic dermatitis $(13,14)$. Recent reports on melanoma tumor models have evidenced that Th9 lymphocytes enhance the intra-tumor expression of chemokines and their receptors such as CCL20 and CCR6, thus promoting the recruitment of dendritic cells and anti-tumor CD8+ T lymphocytes (15).

The role of Th9 cells in rheumatic diseases is less characterized. According to some 
studies, IL-9 may contemporarily favor the differentiation of Th17, acting like IL-6 or IL-21 in the presence of TGF $\beta$, and inhibit the apoptosis of Treg cells, by enhancing the expression of STAT5. Mice knock-out for $I L-9 R$ gene develop a more aggressive form of experimental autoimmune encephalomyelitis when compared to wild types, mainly due to an over-expansion of Th1 lineage (16). Similar results were obtained in a recent report that analysed the cytokine interplay in human skin samples and murine models of atopic dermatitis, finding an indirect regulation of IL-9 on IFN $\gamma$ expression (17). On the other hand IL-23 and IL-21 represent inhibiting cytokines for the development of Th9 lineage. IL-9 mRNA and IL-9R have been found significantly increased in gut specimens from ulcerative colitis patients, where this cytokine may activate neutrophils and epithelial cells, delaying ulcers healing (18). In the gut, Th9 cells are involved in the defence against parasitic infections and an increase in this $\mathrm{T}$ helper subset may represent a link between dysbiosis and autoimmune inflammatory bowel diseases (11). One study on connective tissue diseases reported that IL-9 serum levels were increased in systemic sclerosis but not in systemic lupus erythematosus or dermatomyositis, being inversely correlated with the degree of lung fibrosis (19). This result may be interpreted in the light of the pleiotropic effects of IL9, promoting at the same time the expansion of Treg and Th17 lymphocytes (Figure 2) (20).

To our knowledge, there are actually few studies on the involvement of IL-9 in RA. One study analysed by multiple suspension array the serum cytokine profile of 6 RA patients undergoing rituximab treatment after failing a previous therapy line with infliximab. The authors found increased levels of IL-9 at baseline and at follow-up times in responder patients and concluded that IL-9 may be considered as a predictive marker of response to rituximab (21). Another

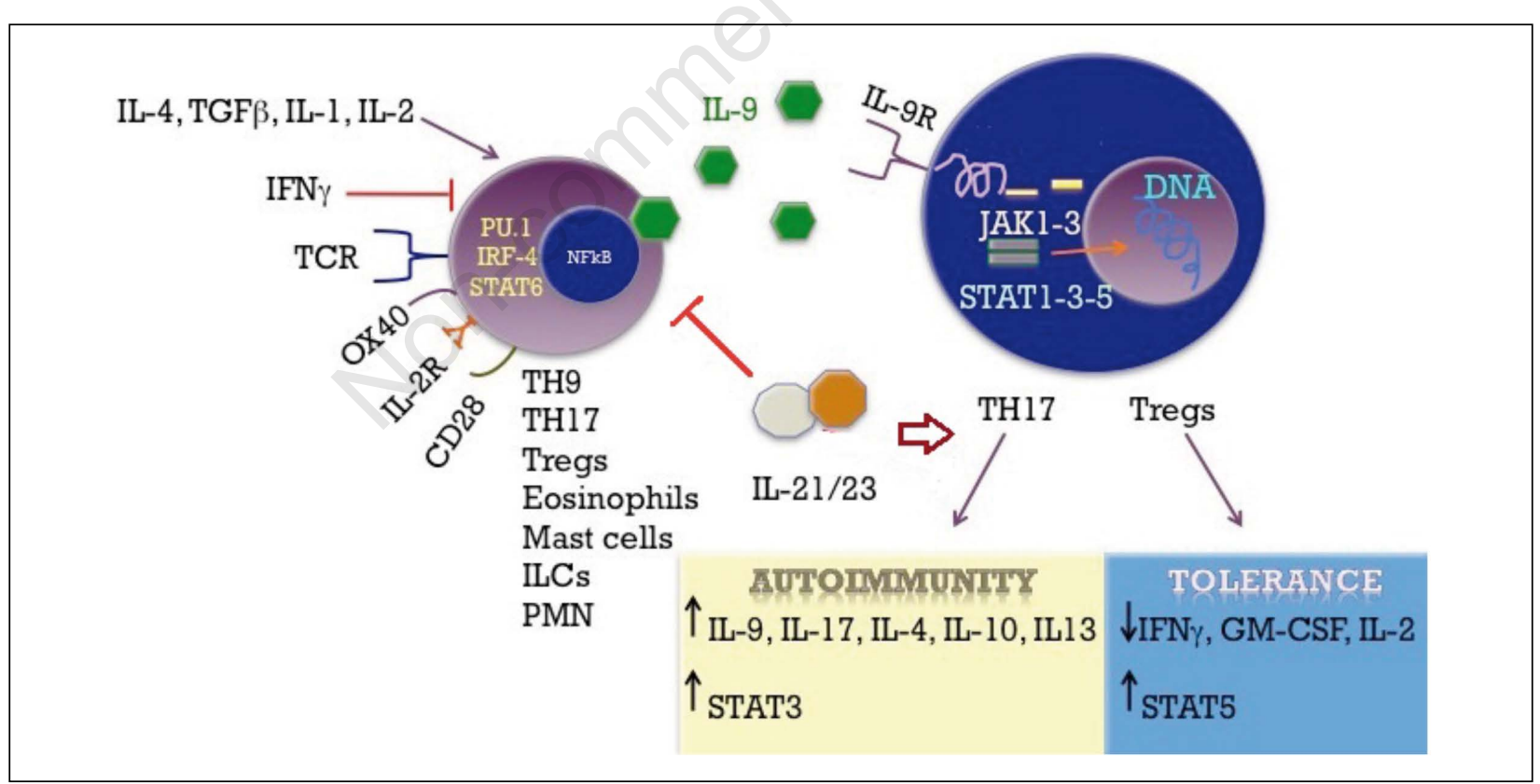

Figure 2 - The network of interleukin (IL)-9 and IL-9 producing cells. Th9 lymphocytes are induced following the stimulation with IL-4, TGF $\beta$, IL-1, IL-2 and the interaction of TCR, OX40 and CD28 with their ligandos; on the contrary, IFN $\gamma$, IL-21 and IL-23 inhibit their differentiation. Th9 cells express specific transcriptional factors (PU.1 and IRF-4) that favor the activation of NFkB. Beyond Th9 lymphocytes, Th17 cells, T regulatory cells, eosinophils, mast cells, innate lymphoid cells (ILCs) and polymorphonucleates (PMN) may produce IL-9. IL-9 interacts with its receptor (IL-9R), composed by a specific $\alpha$ - and a common $\gamma$ chain, thus activating an intracellular pathway that promotes the transcription of specific genes involved in survival of either Th17 (implied in autoimmunity) and T regulatory cells (implied in immune-tolerance). 
study on 44 subjects showed an increased serum concentration of IL-9 in first-degree relatives of RA patients, which was associated to a higher ratio of auto-antibodies positivity, such as RF or ACPAs (22). A recent report on RA, psoriatic arthritis (PsA) and osteoarthritis (OA) patients showed an augmented concentration of IL-9 in blood and synovial fluid of RA and PsA subjects than in OA ones. Furthermore the treatment of magnetically sorted synovial and blood CD3+ T cells with recombinant IL-9 induced their expansion only in RA and PsA samples, underlining the hyper-expression of $I L-9 R$ on cells coming from patients suffering from autoimmune arthritis (23). Finally, a recent histological study on synovial membrane biopsies from RA patients evidenced an augmented expression of Th9 lymphocytes in the most aggressive forms of disease and an association between these cells and the organisation of the lymphoid centres or the synthesis of ACPAs (3). In line with these data, our results showed an increased percentage of Th9 cells in the peripheral blood of RA patients when compared to healthy controls, but no difference was noticed before and after the incubation of PBMCs in vitro with infliximab. A limit of the study was that we did not sorted Th9 lymphocytes according to the specific transcriptional factor profile (PU.1 or IRF-4), perhaps including other T CD4+ subsets in the pool of Th9 producing cells.

However, these data may also reflect the bipolar effects of these cells, favoring at the same time the survival and the activation of Th17 and Treg lymphocytes. Due to their ambivalent behavior, Th9 cells would be therefore not suitable for predicting the response or the failure to a specific treatment in RA patients. Figure 2 resumes the complexity of the role of Th9 cells played in the immune network.

\section{CONCLUSIONS}

In conclusion, IL-9 levels are increased in RA patients. Th9 cells are the major producers of IL-9, and their amount is higher in RA patients than in healthy subjects; however our experiment in vitro does not demonstrate an association between Th9 lymphocytes and the response to infliximab. IL-9 producing cells restore a balance between tolerance and inflammation and, according to our results, cannot be considered a reliable marker in predicting the response to anti-TNF agents. However our study has some limits and the actual knowledge about the biologic effects of these cells in RA is still incomplete and requires further investigations.

Conflict of interest: the authors declare no potential conflict of interest.

Acknowledgements: we acknowledge all of the physicians in the Rheumatology Unit of Luigi Sacco University Hospital for giving us access to the patients' source documents.

Conference presentation: LII SIR Congress, 2015.

\section{$R=E=R E N C E S$}

1. Li P, Spolski R, Liao W, Leonard WJ. Complex interaction of transcription factors in mediating cytokine biology in $\mathrm{T}$ cells. Immun Rev. 2014; 261: 141-56.

2. Alunno A, Manetti M, Caterbi S, et al. Altered immunoregulation in rheumatoid arthritis: the role of regulatory $\mathrm{T}$ cells and proinflammatory Th17 cells and therapeutic implications. Mediators Inflamm. 2015; 2015: 751-93.

3. Ciccia F, Guggino G, Rizzo A, et al. Potential involvement of IL-9 and Th9 cells in the pathogenesis of rheumatoid arthritis. Rheumatology (Oxford). 2015; 54: 2264-72.

4. Talotta R, Berzi A, Atzeni F, et al. Paradoxical expansion of Th1 and Th17 lymphocytes in rheumatoid arthritis following infliximab treatment: a possible explanation for a lack of clinical response. J Clin Immunol. 2015; 35 : 550-7.

5. Aletaha D, Neogi T, Silman AJ, et al. 2010 Rheumatoid arthritis classification criteria: an American College of Rheumatology/European League Against Rheumatism collaborative initiative. Arthritis Rheum. 2010; 62: 2569-81.

6. Schlapbach C, Gehad A, Yang C, et al. Human TH9 cells are skin-tropic and have autocrine and paracrine proinflammatory capacity. Sci Transl Med. 2014; 6: 219ra8.

7. Clair EW, Wagner CL, Fasanmade AA, et al. The relationship of serum infliximab concen- 
trations to clinical improvement in rheumatoid arthritis: results from ATTRACT, a multicenter, randomized, double-blind, placebocontrolled trial. Arthritis Rheum. 2002; 46: 1451-9.

8. Zhao P, Xiao X, Ghobrial RM, Li XC. IL-9 and Th9 cells: progress and challenges. Int Immunol. 2013; 25: 547-51.

9. Dardalhon V, Awasthi A, Kwon H, et al. IL-4 inhibits TGF- $\beta$-induced Foxp $3+\mathrm{T}$ cells and, together with TGF- $\beta$, generates IL-9+ IL-10+ Foxp3(-) effector T cells. Nat Immunol. 2008; 9: $1347-55$.

10. Schmitt E, Klein M, Bopp T. Th9 cells, new players in adaptive immunity. Trends Immunol. 2014; 35: 61-8.

11. Licona-Limón P, Henao-Mejia J, Temann AU, et al. Th9 cells drive host immunity against gastrointestinal worm infection. Immunity. 2013; 39: 744-57.

12. Kaplan MH. Th9 cells: differentiation and disease. Immunol Rev. 2013; 252: 104-15.

13. Übel C, Graser A, Koch S, et al. Role of Tyk-2 in Th9 and Th17 cells in allergic asthma. Sci Rep. 2014; 4: 58-65.

14. Ma L, Xue HB, Guan XH, et al. Possible pathogenic role of T helper type 9 cells and interleukin (IL)-9 in atopic dermatitis. Clin Exp Immunol. 2013; 175: 25-31.

15. Lu Y, Yi Q. Utilizing Th9 cells as a novel therapeutic strategy for malignancies. Oncoimmunology. 2013; 2: e23084.

16. Elyaman W, Bradshaw E, Uyttenhove C, et al. IL-9 induces differentiation of Th17 cells and enhances function of FoxP3+ natural regulatory T cells. PNAS. 2009; 106: 12885-90.

17. Liu J, Harberts E, Tammaro A, et al. IL-9 regulates allergen-specific Th1 responses in allergic contact dermatitis. J Invest Dermatol. 2014; 134: 1903-11.

18. Nalleweg N, Chiriac MT, Podstawa E, et al. IL-9 and its receptor are predominantly involved in the pathogenesis of UC. Gut. 2015; 64: 743-55.

19. Yanaba K, Yoshizaki A, Asano Y, et al. Serum interleukin-9 levels are increased in patients with systemic sclerosis: association with lower frequency and severity of pulmonary fibrosis. J Rheumatol. 2011; 38: 2193-7.

20. Leng RX, Pan HF, Ye DQ, Xu Y. Potential role of IL-9 in the pathogenesis of systemic lupus erythematosus. Am J Clin Exp Immunol. 2012; 1: 28-32.

21. Khan IH, Krishnan VV, Ziman M, et al. Comparison of multiplex suspension array largepanel kits for profiling cytokines and chemokines in rheumatoid arthritis patients. Clin Cytometry. 2009; 76B: 159-68.

22. Hughes-Austin JM, Deane KD, Derber LA, et al. Multiple cytokines and chemokines are associated with rheumatoid arthritis-related autoimmunity in first-degree relatives without rheumatoid arthritis: Studies of the Aetiology of Rheumatoid Arthritis (SERA). Ann Rheum Dis. 2013; 72: 901-7.

23. Raychaudhuri S, Mitra A, Mitra AD, et al. Th9 cells in inflammatory cascades of autoimmune arthritis. Arthritis Rheum. ACR abstracts. 2014; 66: S708. 